\title{
UK PharmSci 2010: The Science of Medicines
}

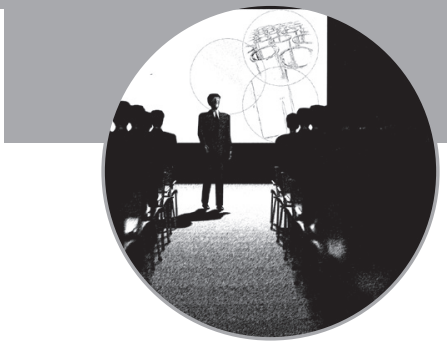

\author{
UK PharmSci 2010: The Science of Medicines \\ I-3 September 2010, Nottingham, UK
}

This conference was billed as the "premier conference for pharmaceutical scientists in the UK in 2010 and was organized by the Academy of Pharmaceutical Sciences of Great Britain (APSGB). It was, in fact, the inaugural annual conference of the APSGB and representatives of both academic and industrial institutions were organized to showcase the "best of pharmaceutical science from the UK and around the world'. A diverse program over 3 days was split into four parallel sessions covering areas such as therapeutics, drug delivery, drug analysis, delivery technology, regenerative medicine, medicinal chemistry and quality by design. Content amounted to a total of I43 podium presentations, 54 by invited speakers and over 90 from selected abstract submissions. Daily poster sessions highlighting 300 separate pieces of original research encircled a trade exhibition by pharmaceutical companies, instrument manufacturers and professional bodies. There appeared to be a consensus that UK PharmSci 2010 was, for 3 days, a worthy forum for the 709 registered delegates based on the feedback given during, and immediately after the event.

UK PharmSci 2010 was a bold break by the Academy of Pharmaceutical Sciences of Great Britain (APSGB) from the tradition of hosting this annual event in conjunction with the Royal Pharmaceutical Society of Great Britain, with which there has been a partnership agreement since the APSGB was founded in 2000. Over the past 10 years, the larger British Pharmaceutical Conference has featured separate programs for both pharmaceutical scientists and practicing pharmacists. Therefore, this year's conference provided a golden opportunity, to permit this organization, which represents scientists working in the pharmaceutical sciences, to focus on the scientific issues that interest the bulk of its members.

Conference Chair, Kevin Shakesheff (University of Nottingham, UK) and APSGB Chair, Eddie French (Pfizer) officially opened the conference with the promise of a varied and full program, evident to delegates from the substantial conference pack received at registration. A fully detailed program was organized into rather fetching and professionally produced pamphlets for each conference day. A full delegate list, exhibition and poster guide were also included with the obligatory notebook and pen. All conference abstracts were of the same format and approximating to one per page of a special issue of the Journal of Pharmacy and Pharmacology [1].

To make sense of the incredibly large and varied program on offer, a snapshot of each day's proceedings are presented.
The main themes on the first day were highlighted as materials science, biotherapeutics, analysis of drug-inclusion products, topical delivery and anti-infectives. For each theme, a combination of invited speakers, selected abstracts and 'poster bites' were arranged at the behest of appointed session chairs. Chairs were composed of a cross-section of respected industrial, academic and consultant pharmaceutical scientists from the UK.

Materials science sessions were subdivided into three streams covering crystal and particle engineering, inhalation and process engineering. Invited speakers covered topics such as the use of pharmaceutical co-crystals to modify physicochemical and dissolution profiles of active pharmaceutical ingredients (Ashwini Nangia, University of Hyderabad, India), innovative methods of engineering particles for inhalation (Peter York, University of Bradford, UK) and the use of combination and triple drug therapies in pulmonary delivery (David Hipkiss, Prosonix Ltd, Oxford, UK). These general lectures paved the way for short presentations based on outstanding abstract submissions on a diverse range of subjects from the effects of speciation in freeze-drying (Richard Storey, AstraZeneca) to the use of dry powders for the orphan disease cystinosis (Barbara Buchan, Robert Gordon University, UK). The former short presentation highlighted the importance of a clear understanding of the critical thermal properties of freeze-concentrated drug/salt compositions for

\section{Kerr H Matthews}

School of Pharmacy \& Life Sciences, Robert Gordon University, Schoolhill, Aberdeen, UK

Tel.: +44 I224 262525

Fax: +44 I224 262555

E-mail: k.h.matthews@rgu.ac.uk 
maintaining porosity in lyophilized formulations, the latter on the potential of delivering noxious aminothiol compounds with none of the usual side effects of nausea and vomiting.

The formulation of delivery systems for biotherapeutics, especially those concerning large molecules, formed the basis of this significant conference theme. An overview of process development strategies for novel antibody formats (Ahmed Yasin, GlaxoSmithKline [GSK]) preceded a presentation on development methods for the detection of protein aggregation (Oliver Croad, University of Nottingham, UK). The need for new analytical approaches to overcome the problem of predicting protein aggregation was discussed and highlighted the propensity of atomic force microscopy to detect the temperature-induced unfolding of proteins.

Another talk highlighted the use of longchain fatty acid amides of spermine to deliver siRNA efficiently for gene silencing (Abdelkader Metwally, University of Bath, UK) even in the presence of fetal calf serum. Production of pharmaceutical microparticles as carriers was covered by John Mitchell (University of Greenwich, UK) who discussed the preparation of solid core drug-delivery systems by supercritical fluid $\left(\mathrm{CO}_{2}\right)$ technology using a model silica core with a fatty acid shell, and Mark Whitaker (University of Nottingham, UK) on the use of supercritical methods for producing the 'perfect injection'. This presentation contained 2D simulations and 3D modeling of poly(lactic-co-glycolic acid) microparticles passing through different needle gauges, determining the limits for blockages.

Sessions on drug inclusion products began with an entertaining presentation by Yvonne Perrie (University of Aston, UK) on the characteristics of different inclusion systems for various routes of administration that included micelles, liposomes and cyclodextrins. An authoritative talk focused on formulation development and the design of delivery systems with particular release characteristics. This neatly set the scene for the following speaker, Abigail Moran (of the Medicines and Healthcare products Regulatory Agency), who highlighted current challenges of licensing drug inclusion products from the medicine regulator's perspective. Micelle-containing products were discussed in terms of regulatory requirements and a long list of physicochemical parameters, including solubility and partitioning of drugs and surfactants on storage, were discussed. It was noted that there is very limited regulatory experience of liposomebased delivery systems and that they are not yet covered by bioequivalence guidelines. Examples of current regulatory challenges in relation to drug-inclusion products were considered.

Under the theme of anti-infectives, quorum sensing (Paul Williams, University of Nottingham, UK) and bacteriophages for antimicrobial chemotherapy (David Harper, BioControl Ltd, UK) formed the subjects of invited talks as did the use of bacteriophages for control of biofilms on implanted medical devices (Geoff Hanlon, University of Brighton, UK). Clearly, alternatives to conventional antibacterial agents are required to tackle the ever-increasing problem of multidrug-resistant pathogens.

The second day of UK PharmSci 2010 was divided into pharmaceutical technologies, regenerative medicine and medicinal chemistry. Chaired by Don Cairns (Robert Gordon University, UK) medicinal chemistry was given a fair hearing at what is not traditionally the main forum for medicinal chemists. Minor groove binders as therapeutic agents (Simon Mackay, University of Strathclyde, UK) and the application of artificial intelligence and advanced statistics to the prediction of the bioactivity of complex natural products (Jose Prieto, University of London, UK) gave a good insight into the modern application of computational chemistry and some of the tools employed to advance our understanding of the complexities of protein-drug interactions and the mechanism of drug action. Submitted abstracts and selected presentations covered a range of subjects that included synthetic 2,4,5-trisubstituted pyrimidine derivatives as powerful cyclin-dependent kinase 9 inhibitors in human cancer cells (Shenua Shi, University of Nottingham, UK), pegylated-cysteamine compounds for the treatment of cystinosis (Graeme Kay, Robert Gordon University, UK) and an update on the use of iron chelation therapy in blood-borne and neurodegenerative disorders (Robert Hider, King's College London, UK). Remarkably, half of all published abstracts in medicinal chemistry originated from three institutions in Scotland, with Sabbir Ahmed's group at the University of the West of Scotland being particularly well represented.

One of the two larger themes, pharmaceutical technologies, encompassed the application of nanotechnologies in cancer treatment, gene therapy, pediatric medicines and general pharmaceutics. Anticancer nanomedicines were expertly covered by Andreas Schatzlein (University of London, UK) and bio-inspired vectors for gene delivery by Helen McCarthy (Queen's University, Belfast, Northern Ireland). One of the foremost experts in 
pediatric medicines, Catherine Tuleu (University of London, UK) spoke about the integrated aspects of formulation issues associated with children's medicines, covering regulation, practice and industrial initiatives. John Hempenstall (GSK) and Jenny Walsh (AstraZeneca) jointly considered formulation strategies for pediatric clinical studies and bringing medicines to market.

Regenerative medicine was split into two sections, namely cell therapies, and drug discovery and stem cells. John Sinden (Reneuron Group Plc) covered the UK regulatory pathway for stem cell therapies while Nick Thomas (GE Healthcare) spoke of the therapeutic and research potential of human stem cells. Clearly, human embryonic stem cells are the most controversial in medical science unlike adult stem cells or pluripotent stem cells [2]. James Dixon (University of Nottingham, UK) highlighted work to test whether a combination of cardiac-specific transcription factors could directly produce induced cardiomyocytes from pluripotent human embryonic stem cells. A combination of four genes was identified that rapidly and efficiently induced the differentiation of human embryonic stem cells into functional beating induced cardiomyocytes in vitro with massive increases (>1000-fold enhancement) in cardiac differentiation over embryoid body methods. The plan is to test these factors with human somatic cells in order to convert them directly to induced cardiomyocytes with a view to produce functional tissue constructs.

A separate session organized by the New Scientist Focus group of the APSGB considered the use of gold in medicine (Trevor Keel, World Gold Council) and modification of bacterial bile acids (Brian Jones, University of Brighton, UK) for potential pharmaceutical application. Another presentation considered the oral delivery of live bacterial vaccines (LBVs) using bile absorbing resins, which were reported to release up to 4000 -fold more live bacteria into bile than conventional dry, powdered LBVs. This was followed by the 'GSK Emerging Scientist Plenary Lecture Award' given to Stuart Jones (King's College London, UK) who eloquently presented aspects of his research into dynamic aerosols for pulmonary delivery using beclomethasone dipropionate as a model. His results suggested

\section{Bibliography}

Papers of special note have been highlighted as:

- of interest

1 J. Pharm. Pharmacol. 62(10), 1201-1516 (2010)

- Conference abstracts are published as a complete volume of J. Pharm. Pharmacol. that $51 \%$ of the drug was present in the lungs, $31 \%$ in the mouth and $18 \%$ exhaled. A general talk on the barriers to drug delivery was given.

Another individual session showcased a selection of the best short papers from a diverse range of submitted abstracts in drug delivery. An alternative to this was offered in the form of an interactive debate with a panel of distinguished academics and industrialists from the three big pharmaceutical companies: Pfizer, GSK and AstraZeneca. Typical questions addressed the key challenges and funding in UK pharmaceutical science. A plenary lecture by the distinguished Professor Trevor Jones CBE entitled, 'Personalized medicine. Opportunities and challenges' rounded off the second day.

The final day was an eclectic collection of parallel sessions covering quality by design issues, interdisciplinary science and more short talks from selected abstracts. A special discussion forum, hosted by the 'Research Councils UK Science Bridges with China and India Program', also featured. The 'Science Bridges' are led by the University of Bradford, UK and the University of Nottingham, UK and are stimulating new collaborations between scientists in the three stated countries. This forum debated the opportunities and challenges of the rapid expansion of pharmaceutical research, development and manufacturing in China and India, highlighting business opportunities. On this encouraging note, the conference officially ended.

\section{Future plans}

Such was the apparent success of this inaugural conference that the APSGB has agreed to organize next year's meeting of predominantly UK pharmaceutical scientists in a similar fashion at the same venue.

Financial \& competing interests disclosure

The author has no relevant affliations or financial involvement with any organization or entity with a financial interest in or financial conflict with the subject matter or materials discussed in the manuscript. This includes employment, consultancies, honoraria, stock ownership or options, expert testimony, grants or patents received or pending, or royalties. No writing assistance was utilized in the production of this manuscript.

2 Sleigh SH, Barton CL. Realising the potential of therapeutic stem cells with effective delivery. Therapeutic Delivery 1(1), 11-15 (2010). 